\title{
How Potential New Members Approach an Online Community
}

\author{
Janghee Cho ${ }^{1, *}$ (1) \& Rick Wash ${ }^{2}$ \\ ${ }^{1}$ University of Colorado Boulder, Boulder, CO, USA (E-mail: janghee.cho@colorado.edu); \\ ${ }^{2}$ Michigan State University, East Lansing, MI, USA (E-mail: wash@msu.edu)
}

Accepted: 9 November 2020

\begin{abstract}
Online communities, socio-technical systems where people interact with others, depend on new members coming to the community. While the majority of research in online communities relation to the recruitment of new members has focused on new members' socialization and retention, little work has focused on how potential new members who are not yet a member of the community make the decision on whether they are willing to join in the online community. To understand this initial decision process, we investigated how potential new members build mental models of the online community from their first experience within the community, and how this process impacts the decision to continue participating in the community. We interviewed 31 potential new members of the online communities, Quora and Reddit, to better understand how they evaluate a new community. We found that the process of understanding a community involves orienting toward multiple different aspects of the community, including the content available on the community, the people who are already part of the community, and the technology interface and mechanisms that control the community. Participants who focused on consuming and enjoying content were much more likely to express an interest in future participation in these communities than participants who just evaluated the community, looking at the people in the community or the technology of the community. This extends previous considerations for recruiting new members in online communities. We conclude by discussing how our findings can have broad implications in developing successful online communities and suggesting future research efforts that could help understand potential new members.
\end{abstract}

Key words: CSCW, Joining, Legitimate peripheral participation, Mental models, Online communities, Recruiting

\section{Introduction}

Online communities are groups of people who interact with each other, mediated by technology such as computers and the Internet. They allow people to coordinate and produce at a scale impossible without the Internet. Even though each online community has different goals and purposes of existence, every online community has one thing in common that needs to be considered in order to 
maintain it: recruiting new members. This is because online communities depend on volunteer activity - people coming to the community and deciding to participate. Not everyone who hears about a community decides to participate, but communities depend on the people who do. However, that decision to participate is complicated. Before making that decision, people need to develop an understanding of what that community is, how it works, and what it means for them to join, and then make a decision about whether they want to participate.

An online community is a socio-technical system. Each online community can be considered to have three major components (Kraut and Resnick 2012): 1) one or more technologies - often a website running some forum or other interactive web-based technology - that enables communication between community members and has a specific set of affordances, such as content archiving, search capabilities, or member profiles that shape the interactions; 2) a specific group of people who participate in the community and who form the social basis for the community; and 3) a set of content that already exists in the community that is available to community members (and often, the public). Each community may choose to emphasize one component more than others, but all online communities include all three components and these components interact in complicated ways to produce the final community, which is explicitly or implicitly exposed to new potential members.

Much research has investigated how these components can be leveraged to recruit new members and make them engage with an online community (Kraut and Resnick 2012). This process is often viewed through the lens of legitimate peripheral participation, which is a transformational process based on socially situated learning (Lave and Wenger 1991). It usually serves as a theoretical ground to shed light on how newcomers or passive consumers become active members in online communities research (Bryant et al. 2005; Antin and Cheshire 2010; Fiesler et al. 2017; Lampe and Johnston 2005; Mugar et al. 2014). For instance, Bryant et al. (2005) found that newcomers become members by participating in less important activities or observing old-timers' artifacts or communities of knowledge and practice. However, most previous research focused on how to socialize new members (e.g., help them learn how to behave in ways appropriate to the group) or how to motivate them to participate in activities. Although much research has focused on these newcomer's integration processes, we know very little about how potential new members who haven't yet decided if they will join the community become legitimate peripheral participants who are often seen as a new or passive member in the online community. Potential new members are not defined as individuals who presumably belong to an online community, but individuals who have no direct experience within the online community and have potential to join as a new member. These potential new members' decision process is critical because online communities must attract and motivate enough people 
to join the community in order to sustain it. "If you build it, they will come"1 isn't true for online communities, and is a common fallacy that frequently leads to failed communities. To overcome this challenge and better understand how the decision to participate in the community is made, our study focuses on a phase in which potential new members have their first experience by interacting with socio-technical components in the community rather than a phase in which new or inactive members become engaged in the community.

In deciding whether to join a community, we suspected that people try to understand the community by forming a mental model of it. Mental models are representations based on reality as a person experiences it and are used to help people make choices about how to behave. Mental models naturally change and develop over time, adapting to new information and new experiences (Jones et al. 2011). When a person first approaches a new online community, there are many different ways they can begin to think about that community: who is in the community, what is the community about, and most importantly, what is likely to happen if I participate in the community? The notion of legitimate peripheral participation has a limitation on understanding how a person's first understanding is formed because potential new members are not considered as an actual member in an online community. So their interactions with the online community cannot be seen as socially situated learning, grounded on socio-cultural understandings of practices. So, in this paper, we focus on initial mental models - the first understanding formed about an online community - to identify how new potential members investigate an online community from their point of view. People use this mental model to develop expectations about the future of the community. These expectations are critical in both the decision to participate and in understanding the community's reaction to participation.

We conducted an interview study to elicit users' perceptions and expectations on the online communities - Quora and Reddit. We asked participants to thinkaloud while browsing the community. We focused on how they describe their thoughts and feelings and form expectations about future participation. We identify that potential members go through a distinct evaluation stage where they evaluate the online community by successively focusing on different aspects of it. The contributions of this paper are twofold. First, we provide empirical findings of how potential new members evaluate online communities to make a decision whether to join a community. The majority of attention is focused on the existing content of an online community, though time is also spent looking at how the community functions (the technology) and the social relationships that exist (the people). Second, we discuss how this evaluation process is an in-between process, with the person neither as a full participant in the community nor as an intentional

${ }^{1}$ Dir. Phil Alden Robinson. Field of Dreams. Gordon Company, 1989. Kevin Costner and James Earl Jones. 
non-user of the community. We identify the need to design for this in-between "use" of the community.

\section{Related work}

Kraut and Resnick (2012) identify 5 major challenges that all online communities face: (1) motivating contribution, (2) encouraging commitment, (3) regulating behavior, (4) recruiting new members, and (5) achieving critical mass. In this paper, we focus specifically on the fourth problem: online communities need to find, recruit, and socialize new users to prevent the community from whithering away with the natural attrition and turnover that happens.

Most online communities face a natural member attrition: members of the community will eventually stop participating in the community (Velasquez et al. 2014). Butler (2001) analyzed listserv-based online communities to examine the factors that lead people to join the community or leave the community. He found that often the same factors, including volume of communication and variation in content, both lead people to leave the community and lead others to join. This result effectively describes turnover, which is when some community members leave the community and at the same time, as others join the community, and is a natural process for communities. To combat this attrition, online communities must be constantly seeking out new members to join the community. Even though an online community already achieved a critical mass of active participants, it is necessary to keep recruiting new members to be successful.

\subsection{Recruiting new members in online communities}

Kraut and Resnick (2012) identify five challenges that online communities face as they seek new members: 1) recruiting, 2) selecting, 3) retaining, 4) socializing new members, and 5) protecting the community from newcomers. To overcome these challenges, much research in $\mathrm{HCI}$ and $\mathrm{CSCW}$ has been examined to understand how users' experiences and behaviors look like in this recruitment phase and how online communities should be designed and improved to successfully recruit new active members. While some studies are conducted from the perspective of the online community (e.g. Lu and Farzan, 2015, Lu et al. 2017; Burke et al., 2009), this study considers the narrative from the point of view from individuals who may see a different set of challenges from their point of view.

There are multiple theories about how new or inactive members in online communities changes over time. In studying the online geowiki Cyclopath (Priedhorsky and Masli 2010), Panciera et al. (2010) found that the vast majority of members who would ever contribute began doing so on the first day, while the majority of people who lurked did so on the first day. Finding a similar pattern in Wikipedia, Panciera, Halfaker, and Terveen argue that people are born into roles in an online community when they first join, rather than evolving into roles (Panciera 
et al. 2009). Contrary to this Born-not-Made approach, the other major theory is the Reader-to-Leader framework (Preece and Ben Schneiderman 2009). In this theory, newcomers participate in relatively less important activities (e.g., reading contents and following the community), learning much about the community, including community norms, options of participation, and what types of contributions are valued. Some, but not all, members then move on to future roles in the community by contributing content. Possibly, after being a contributor for a while, they may move on to other leadership roles in the community.

The notion of legitimate peripheral participation, coined by Lave and Wenger (1991), has been considered a theoretical lens to understand this Reader-to-Leader process of how newcomers become engaged in a community. Lave and Wenger (1991) introduced a novel idea of learning: rather than looking to learning as the acquisition of knowledge, people gain knowledge through a process of social participation in a community of practice. In this social learning approach, new members are situated in a learning process by participating in legitimate peripheral activities. Although a conceptual idea of the community in Lave and Wenger (1991)'s framework connotes a broader sense of the group in organizational persepctive, online communities have been considered a practical exemplification for legitimate perpheral participation (e.g. Johnson 2001; Bryant et al., 2005; Fiesler et al. 2017). For instance, Jackson et al. (2016) analyzed new members of the citizen science community Zooniverse, and found that new members initial sessions can be clustered into six categories. The majority of new members focused on light work that involves simple and brief browsing; other new members would engage in intense viewing and contributing, careful annotation of existing content, simultaneously talking with others and annotating, deep viewing, or specializing in a specific type of content (stars). Through these legitimate peripheral activities, new members understand community norms and practices to become more engaged in the joint enterprise.

Most online communities have a large number of people who lurk; they participate in the community by reading and consuming content but never contribute or formally join the community (Nonnecke and Preece 2003). In previous research, legitimate peripheral participation is a lens that helps researchers understand that lurkers are learning about the online communities and may eventually contribute to the online community. Antin and Cheshire (2010) found reading contents helps lurkers understand the mechanism in Wikipedia and to motivate them to participate in more active works. As the framework of legitimate peripheral participation has been applied in a context of online communities, the extent of peripheral activities becomes broader; not just productive tasks, inactive works are also considered as a strategic activity that can contribute in the development of online commuinites (Nonnecke and Preece 2003; Muller 2012). Moreover, the targeted objects for legitimate peripheral participation are not confined to new members, but lurkers who have potentials to become active members. 
The majority of research on new members and lurkers focuses on the retention and socialization phases in which they are considered as a individual who might have already decided to join in the online community or whose data can be traceable. Although Seering et al. (2020) studied first-time participation in an online community by analyzing participants' initial posting behavior, their perspective on the initial engagement is the community's point of view rather than the individual's one. To see what initial posting behaviors are highly related to future retention, they focused on users' first social behavior (i.e., sending a message) rather than users' first experience to visit the community. We believe that there is an important phase that occurs between recruiting (i.e., after the person has heard about the community) and before selection from users' perspective, which is when a potential new member is trying to understand the online community during their first experience within the community. This initial evaluation phase before deciding to join and contribute in the community is critical for users who may want to join an online community, but is under-represented in existing research.

\subsection{Mental models}

Mental models are a simplified understanding of some phenomenon in the world that a person can use to reason about that phenomenon (Jones et al. 2011). Mental models are naturally inaccurate and incomplete (Jones et al. 2011), but contain causal theories about how the world works (Gelman and Legare 2011). A common method of using mental models when placed in a new situation is mental simulation; people use cause-and-effect relationships in mental models to simulate potential future results from actions and decisions to help them decide what action to take (Klein 1998). People also use analogical comparisons with simple or familiar systems to understand new situations or technologies (Eslami et al. 2016). It is natural to understand new knowledge by extrapolating from previous experience or knowledge (Norman 2013).

People frequently form mental models about the technologies that they commonly use. For example, Kempton (1986) studied the mental models that people had of home thermostats, and found two very different mental models that people used to help choose temperature settings. Critically, he argues that mental models of technology do not have to be factually correct to be useful; indeed, his subjects with the less factually accurate model ended up making better decisions.

People also naturally form mental models of people and their motivations. Wash (2010) studied the mental models that home computer users have of computer security threats that they face. He found that many of the mental models were of hackers - of the people who might attack them, including the motivations and capabilities of the attackers.

Since users' mental models guide decision-making regarding the use of technologies (Norman 1983), a number of previous studies have adopted the mental model approach to examine users' understandings of technology and its effect 
on their behavior (Wash 2010; Kang et al. 2015). This mental modal approach resonates with our work that investigates how a potential new member evaluates online communities and makes a decision whether they are willing to join in the community based on their mental models. Online communities are complex sociotechnical systems that include technology components (the website) and people components (the other community members), and the interactions between them. It isn't clear what a mental model of an online community would look like when a potential member encounters it; would it look more like a model of how technology works like that of home thermostats (Kempton 1986)? Would it look more like a model of people and their motivations like those of hackers (Wash 2010)? Or would it include a complex interactions of the two?

\subsection{Non-use of online communities}

Recently, there has been research about reasons why people choose to not use specific technologies. For example, Baumer et al. (2013) surveyed people who had the option to participate in Facebook but chose not to. They found multiple reasons that people cited for not participating, including privacy, concerns over data misuse, concerns about lost productivity, and external pressures. However, they found that for many people, there was not a clear distinction between use and non-use. Similarly, Velasquez et al. (2014) found that people who no longer used Everything2 were really "latent" users, capable of re-engaging but who had chosen to not participate at this time.

Baumer et al. (2015) emphasize that non-use is a choice that is worth studying, and people often have good reasons for choosing to not participate or use technology. Baumer and Brubaker (2017) argue that the metaphor of a technology "user" is not an appropriate metaphor, because there are a wide variety of patterns of interactions with technology that are not captured by the "user" metaphor. For example, some people use technology indirectly through others (e.g. asking someone to Google something for you), have transient interactions with technology, present themselves as multiple users, or who intentionally choose to stop or not participate with a technology.

We argue that potential new members in online communities are in a new, under-studied state that is neither a user nor a non-user. Rather, potential members are in a state of decision-making that exists for a non-trivial amount of time where the person is trying to decide whether they are a user or not. In some ways, they are a user; they are browsing and viewing content much like a lurker might. In some ways, they are not a user; they have not yet consciously chosen to participate in the community. However, they have not chosen to be a non-user like those in study of Baumer et al. (2013); instead they are still in the process of making that decision. Much like (Baumer and Brubaker 2017) argue, we believe that the terms "user", "community member", and "lurker" are not appropriate at 
this point, but these people still have an important relationship with the community and the technology. In this study, we explore how the interaction between an online community and potential new members looks like and examine the notion of participating in the community to extend a discussion of non-use.

\section{Method}

To understand how people make initial participation decisions, it is not sufficient to look at log data from users of an online community. Often, log data only includes interactions after a person has signed up for an account (Preece and Ben Schneiderman 2009), though some research has tracked users before signing up (Panciera et al. 2010). However, this log data misses much of what happens before a person shows up at the website: it misses offline interactions between the potential user and their friends/acquaintances, some of whom might be users of the community. It misses hearing about a community from the news, or overhearing discussions of the community by strangers. And it usually misses how potential users look around and examine the community to figure out if they would like to be part of it.

To address this, we conducted a series of semi-structured interviews with people who were on the verge of joining an online community: people who had heard about the community of interest from other people, may have started to form an impression of the community, but who had not yet visited or joined the community. We interviewed each person to better understand what they had heard (second-hand information) about the community, and then asked them to sit down and use the community for the first time while thinking out loud. The interview protocol is included in the Appendix A. This interview structure allowed us to understand how each person approached the challenge of understanding what a community is and whether they wanted to participate or not - and if so, how to participate.

After considering several platforms, including Facebook, Wikipedia, webMD, and a number of small, geographically-focused online communities, we decided to focus on two communities that were large enough at the time of the study (i.e., 2014) that people would have heard about them, but still small enough that many people had not (yet) joined: Quora and Reddit. Quora had approximately 1 million monthly active users at the end of 2013. ${ }^{2}$ Reddit had about 3 million logged in users in October of 2014. ${ }^{3}$ These communities were ideal for examining how potential new members' initial mental models are built or updated for several reasons. Both of these communities (which each have multiple sub-communities) are very large and growing, and yet many people have just barely heard of them and

\footnotetext{
2 https://quorabot.quora.com/How-many-users-are-on-Quora-really

${ }^{3} \mathrm{http}: / /$ www.redditsecrets.com/how-many-people-use-reddit
} 
have not yet made a decision about whether to join them. So, a substantial number of people might not visit these online communities (i.e., a direct first experience) to form their understandings, and thus have no mental model or a vague one that had been built by external components such as their friends. Additionally, these two communities are not specialized, and have a broad appeal by accumulating all kinds of diverse content from other websites and its sub-communities, known as, for instance, subreddits. Although they are comprised of many subcategories or sub-communities, Reddit and Quora can be considered as one of the information sharing communities for potential new members. Due to this, we believe that, potential participants in our research are likely to be interested in them, no matter how diverse their interests are. These two communities are similar to many other large online communities and social media that cater to diverse types of people, and recruit community members from the general public. We note that our study presents the results now as these two online communities have flourished and become more diverse, and we think that the findings from our study can help to guide the structure and organization of similar, early-stage online communities before they reach mature, global scale.

\subsection{Quora}

Quora (Figure 1) is an online question-and-answer website where community members can post questions to the community and other members will answer them. Quora was launched to the public in June of 2010 (Kincaid 2010), so it had been around for about 4 years at the time of the study. Quora had approximately 1 million monthly active users at the end of 2013. Quora was definitely growing quickly at the time; their monthly active users doubled in the 12 months before the study $^{4}$. Questions on Quora are organized into topics, but these do not form clear subcommunities. Recommender systems in Quora are considered core technology to provide better information. For instance, on the home feed, Quora selects and displays the most interesting contents based on collected data from users (Yang and Amatriain 2016). Wang et al. (2013) found that heterogeneity of interests and Quora's algorithms do a good job of driving user attention toward a relatively small number of popular questions. Wang et al. (2013) also found that Quora has a relatively small number of heavy users that get large amounts of attention, and use that attention to encourage others to answer questions. Paul et al. (2012) found that the credibility of the person doing the answering is very important. Many people also use Quora anonymously. Paskuda and Lewkowicz (2015) found that anonymous users are treated very similarly to people who disclose their identity, including having similar responses, similar comments, and similar politeness.

${ }^{4}$ https://quorabot.quora.com/How-many-users-are-on-Quora-really 


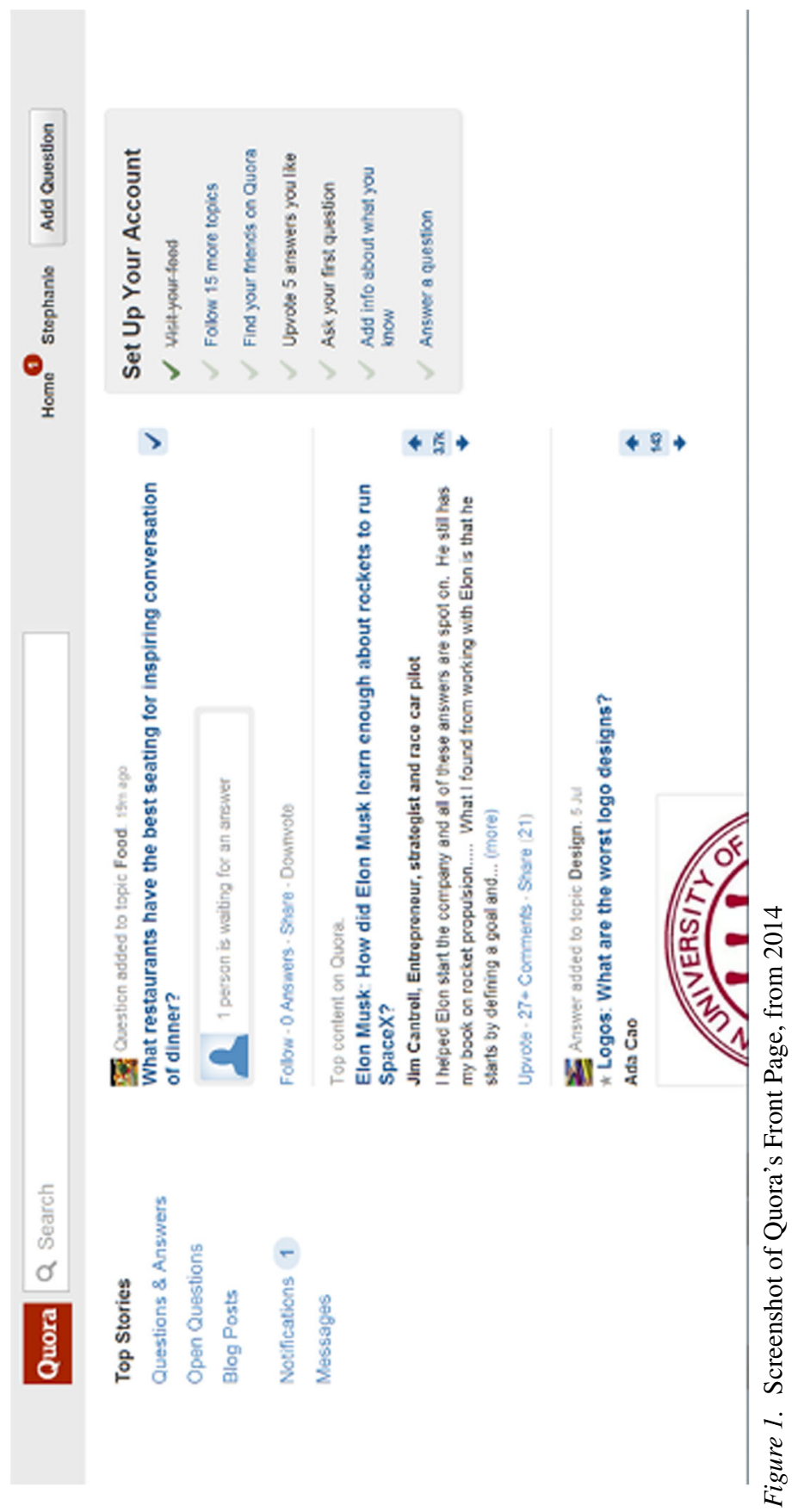




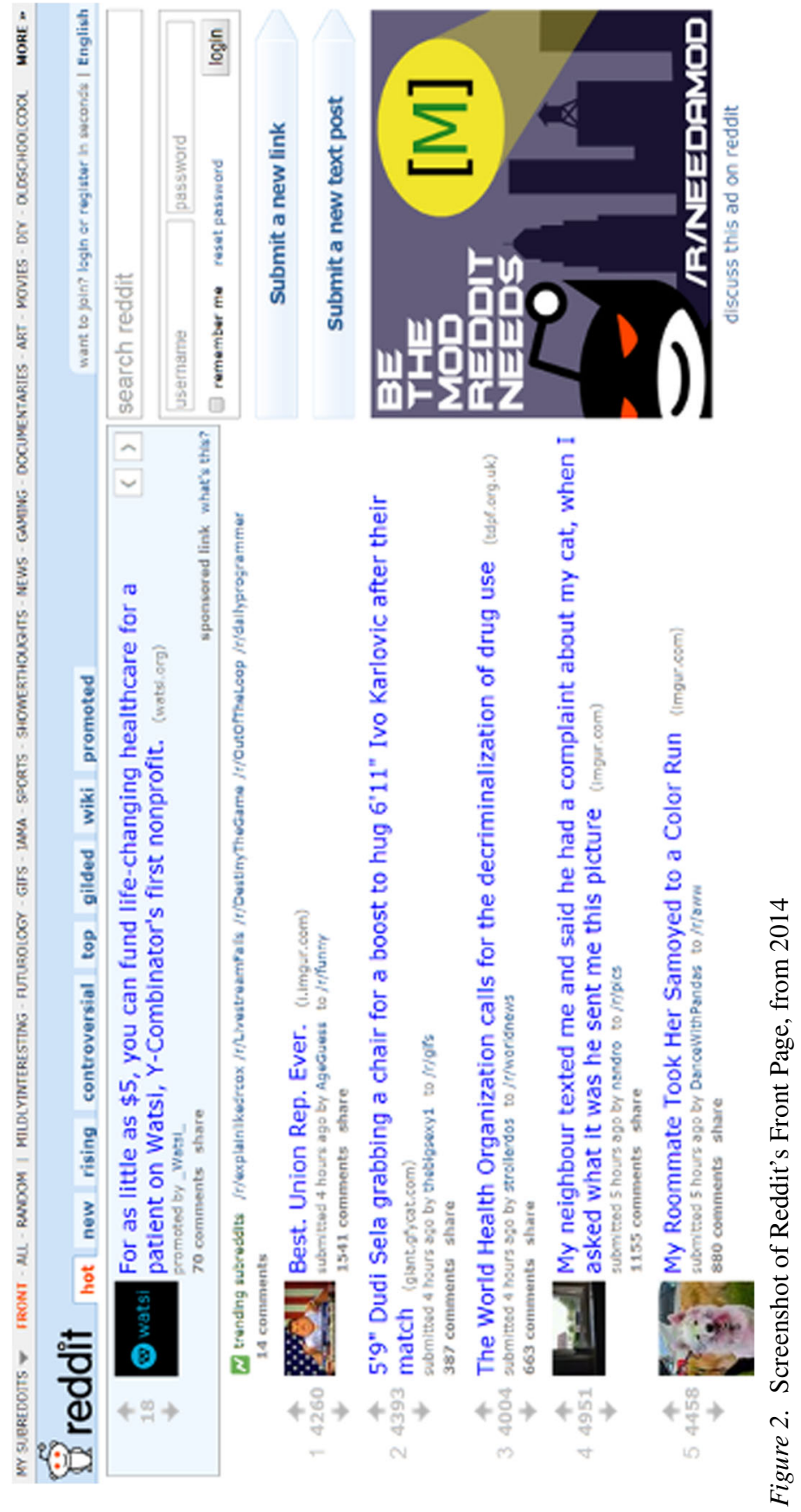




\subsection{Reddit}

Reddit (Figure 2) is an online link aggregation community. Reddit allows members to post a link to an online resource (like a news article). Other members can vote the link up or down (which affects the algorithm that displays and orders links), and can hold a discussion about the links through the commenting system. Reddit had about 3 million logged in users in October of $2014,{ }^{5}$ and has seen rapid growth for a number of years. ${ }^{6}$ Reddit is organized into "subreddits", which are subcommunities inside reddit focused on a specific topic. Weninger et al. (2013) argue that Reddit uses these subreddits to effectively organize news and events into topical hierarchies. Buntain and Golbeck (2014) analyzed user participation in Reddit, and found that most community members only participate in a single subcommunity (subreddit). They also found that most subcommunities have a clear "answer person" that answers questions and interacts with strangers and newcomers, but most regular members are "discussion people" who mostly interact with each other. Choi et al. (2015) showed that Reddit members who are interested in multiple topics play an important role in starting big conversations, while people who post frequently on Reddit are more important for keeping such conversations going.

\subsection{Subjects}

To study potential new members, we recruited people who had heard of one of these two online communities but were not yet active users. We recruited subjects by sending a single email to approximately 5000 students enrolled at a large midwestern R1 state university during the summer of 2014. Students are explicitly in the target population for both communities and are also likely to have heard about but not yet used these communities. And, as people who are interested and engaged in the world, students are reasonable potential members of both communities.

The email included a link to a screening questionnaire that asked, separately for each of Quora and Reddit: 1) whether they had heard about the website; 2) how often they browse the website; and 3) how often they post to the website. Subjects were considered eligible for the study if, for one of the two communities, they answered that they browsed the website less than once a month or less and that they never had posted to the website. While this does not necessarily mean that our participants would have joined the website if we hadn't interviewed them, the fact that they have heard about the website suggests that their social group includes members of the communities.

\footnotetext{
5 http://www.redditsecrets.com/how-many-people-use-reddit

${ }^{6} \mathrm{http}: / /$ redditmetrics.com/history
} 
We had 171 people take the screening survey, and 41 subjects who were eligible for the study. We successfully scheduled and conducted interviews with 34 subjects; the remaining ones did not show up for the interview. Each subject was assigned to be interviewed about the community that they had less experience with (or randomly assigned in the case of ties). 20 subjects were interviewed about Reddit, and 14 were interviewed about Quora. However, we had to remove 2 subjects after being interviewed because they turned out to be more active users than we were looking to speak with and 1 subject due to data loss from a recorder malfunction. Our final analysis was of 31 subjects (Male: 15, Female: 16). We provided more details of our participants' background in Appendix B.

\subsection{Procedure}

The data was collected in the summer of 2014. The interviews were conducted in person in our research lab and lasted at maximum one hour. Subjects were compensated $\$ 15$. Subjects browsing Quora were required to create an account on the site because the website does not allow anonymous browsing ${ }^{7}$; subjects browsing Reddit were not required to do so.

After participants granted consent, we began the interview by asking the participant about the community that we were focusing on for that participant, including questions about what they had previously heard about the community and if they had ever previously visited the community. These were asked first so they wouldn't be biased by actually visiting the community. For the majority of the interview, participants were asked to browse the community and "think out loud." While think-aloud protocols are commonly used in HCI to evaluate the usability of websites, we used it more along the lines that it was originally developed, as a way to understand the details of human problem-solving process and how people are thinking about the tasks they are undertaking (Anders Ericsson and Simon 1980). The think-aloud method has been used to reveal users' mental models of technology (Halasz and Moran 1983; Kang et al. 2015). Asking participants to think aloud enables us to understand the cognition that is happening without substantially changing it (Anders Ericsson and Simon 1980).

\subsection{Analysis}

We followed a process of inductive coding outlined by Miles et al. (2019). We created a summary sheet for each subject immediately after the interview. Audio recordings of the interviews were professionally transcribed, and most of our analysis was of these transcripts. We then used multiple rounds of coding and analysis punctuated by tentative conclusions. We used a bottom-up (inductive)

\footnotetext{
${ }^{7}$ Users with direct links, such as from Google, can anonymously read individual answers but cannot browse the site.
} 
coding process to identify themes and patterns in what the subjects said and did. We summarized these for each subject into a data matrix (Miles et al. 2019). We then built additional intermediate analysis data matrices that summarized specific characteristics of individuals and looked for patterns inductively across individuals. We then tried to assign subjects to "mental models" that represented common patterns across multiple subjects.

Once this was completed, we returned to the data to compare the mental models with the statements and actions in the data to check for representativeness (to ensure that our descriptions represented our subjects well) and to specifically look for contradictory evidence, negative cases, and counter-examples to the models (Onwuegbuzie and Leech 2007).

Our first analysis failed these checks; the descriptions of mental models we identified did not capture participants well and we found multiple negative cases that the models did not explain well and provided contradictory evidence to our tentative results. We went back to the data and identified a new set of themes and facets of the mental model creation process. We re-coded the data for these new themes and facets, and re-built a new set of data matrices and written summaries. This second analysis fared much better and appeared to both capture subject experiences well and we were not able to find negative cases that disagreed with the proposed summaries. The results of this second analysis are presented here.

\subsection{Limitations}

Our goal in this work is to investigate a potential new members' process of understanding a community and their evaluation of the components in the online community. For this, we specifically recruited participants who have little knowledge and experiences with Reddit or Quora. However, it is possible that participants passed our screening but are not really a potential members of the online communities. Since we did not control a degree of motivation to join in these two online communities, our participants might not be interested in joining in them at all. Moreover, participants described their decision whether or not to join a community because the interview was designed to ask about it deliberately (near the end of the interview). Without such a question, they might not have formed a final decision. In addition, since our participants were limited to university students, these demographic characteristics may also influence their evaluation process of online communities. Nevertheless, our findings can be considered evidence about how non-members navigate and evaluate socio-technical components in online communities to continue participating in the community. Also, the final decision might not be representative of the decision the subject would have made absent our study, but the decision-making process is still likely to be similar.

Given our approach that we used a think-aloud qualitative study to navigate online communities, our findings might be influenced by the procedure we designed and the background knowledge participants had. In a naturalistic setting, 
the first page in which people enter the online communities can vary from person to person. For instance, one can visit this community via a link a friend shared, which might be not a front page. Our observation is only one of many ways of navigating online communities and this may influence participants' decisions about whether to join. Considering this limitation, we did not impose any restrictions on navigating the online communities for a think-aloud session, thereby allowing participants to check the communities however they want. The other limitation regarding the think-aloud approach is that evidence in this study is dependent on how participants described their reaction in verbalized words, which may not represent the whole understandings and perceptions of the community they have. Despite this limitation, we believe that the think-aloud approach allows us to investigate how participants describe what they are doing and how they are thinking, thereby interpreting their understandings in a more natural way.

Another limitation of this work is its generalizability to other online communities. Different online communities will vary in terms of a volume or diversity of members, how well it is known, and types of content. It might be difficult to identically apply our findings to the other communities. For the same reason, our findings might no longer be accurate for Reddit and Quora, since data in this study was collected in 2014. These communities are not situated in the early stage of development any more; rather, they are in a more mature stage where most people have used them. Despite of these limitations of generalizability across all types of online communities, our findings from these two online communities are representative of a large online community in the early stage of development. We hope that our empirical findings can be used as a starting point to understand potential new users in the other types of communities.

\section{Findings: what potential new members notice}

We begin by describing what potential new users notice as they are first looking at the online community. These first interactions are critical; they provide the initial observations that potential new members use to form their understanding — their mental model — of the community.

When people are interacting with a complex socio-technical artifact like an online community, they usually orient themselves by focusing on one or more specific aspects of that artifact at a time. This is known as source orientation (Sundar and Nass 2000); when interacting with a complex entity like a computer, there are multiple sources that a person can be oriented toward, and people tend to focus on one possible source. People naturally orient toward sources that feel closer, but any of a set of "re-orientation factors" in the artifact can cause people to focus or reorient to a different source and therefore change the mental model they use and how they engage with the technology (Solomon and Wash 2014). In this section, we describe the patterns we saw in what people successively choose 
to orient toward and develop their understanding as they initially view the online community for the first time.

We discovered that most of our participants would re-orient to different aspects of the community multiple times during their initial session of viewing the community. During these sessions (which usually lasted from 5-10 minutes), we observed our participants re-orienting and changing their focus at least once and up to seven times, though most people changed their focus between twice and four times. We described three different socio-technical aspects in which participants oriented in order to develop their understandings of the communities below.

\subsection{Orienting on content}

The primary feature of these communities that people oriented toward is existing content. All online communities have some form of content, and every one of our participants focused on the content available in the community for at least some portion of the time they spent looking at the site.

In examining the processes that our participants used when focusing on content, we found four different patterns of approaches that were used. At any given time, a participant was only oriented toward one approach, but they might later re-orient themselves to approach content differently.

\subsubsection{Skimming content}

By far the most common orientation was skimming the content of the online community. Participants would browse or skim through the content available on the front page or pages easily reachable. The participants were not taking a closer look at content to become involved or indulge in it; participants just saw content because it was displayed on the website. Rather than carefully or intensively reading content, instead, they were choosing anything that came in sight. Some participants just skimmed a title or a headline to see whether they could find any interesting topics. They found lists of titles of content particularly useful for gaining a big-picture view of the community. They aimed to know whether they are able to find contents related to their interests. 28 out of the 31 participants were oriented toward skimming at least once during their initial viewing of the community.

Participants usually oriented toward skimming near the beginning of the thinkaloud session. As they did this, participants perceived the content as a tool to recognize the online community's purpose, instead of consuming the content directly. As they did this, participants seemed to have two different goals: identifying what the community is about, or unconsciously evaluating the community. As participants skimmed content, their goal was to quickly look over lists of content to try to identify the overall purpose of the community; that is, to understand from 
the content what the content on the community is typically about. For example, as participant $57 \mathrm{R}^{8}$ skimmed through the front page of Reddit, she said:

57R: Yeah. I'm just kinda scanning. But these don't seem that very controversial. So... I don't know why they said that that's kinda misleading. 'Cause I thought it was gonna be more like political topics but...

A second goal that participants had as they skimmed the community was evaluation: they briefly and quickly looked at content to try to evaluate it. While browsing the front page of Quora, participant 7Q said she was trying to see the general type of questions in the community:

7Q: Yeah, just see what people are asking about I guess. Yeah. Like, "What are good ways to prepare my kids to be billionaires?" [...] It was just kind of... I don't know why somebody would ask that.

As they skimmed content, participants would use this evaluation to decide where to navigate to next. Participant $2 \mathrm{R}$ happened to see a video about physical fights between one woman and one man, and then she said:

2R: Whoa. She just flipped him. Oh my gosh. Okay, that's really funny. Alright so, now I'm gonna keep scrolling down Controversial, because that one was pretty entertaining.

\subsubsection{Consuming content}

Separately from skimming, a number of participants spent time explicitly seeking out content for consumption. 21 out of the 31 participants explicitly sought and consumed content. They enjoyed consuming the content itself by talking aloud what they were interested rather than evaluating the quality of community or understanding what the community is. While consuming content, participants intentionally spent their time reading texts or watching video that other existing users left on the community. They usually expressed their excitement about contents (e.g., 'Wow. "When I was in the military, they gave me a medal for killing two men and a discharge for loving one." [reading texts from the community] Oh wow! That's amazing.' (76R)) after finding out interesting content.

Participants initially paid attention to a certain type of content that attracted their awareness, and then became involved with that content. The most common type of content participants in both online communities sought was something fun and entertaining. Many participants looked at the content on Reddit or Quora as a source of entertainment, looking for links or discussions that were in some way fun or entertaining to them. These participants would often discuss visiting online communities or social media while "killing time", looking for content that

\footnotetext{
${ }^{8}$ Participants were randomly assigned numbers to preserve anonymity. These numbers are appended with an ' $\mathrm{R}$ ' for subjects who were interviewed about Reddit or a ' $Q$ ' if they were interviewed about Quora.
} 
matched their interests and provided some amusement. For example, participant 27Q discussed how soccer was one of his interests. As he read content about soccer on Quora, he came upon an question and answer about a recent game:

27Q: This guy is saying he thinks it was a good move to take Rooney ${ }^{9}$ out. I wanna see why. I mean, I agree with him.

However, other participants looked at the content very differently, and explicitly sought out content on a specific topic or small set of topics that they wanted to know more about such as news content or content related to their major. They tended to particularly enjoy knowledgeable content that helped their studies or work. Participant 99Q described how he was 'trying to get interested in my major and learn a little bit more about that, so I can be knowledgeable and be competitive in the workplace' (99Q) and was looking for content that could help him with that. He wasn't evaluating the content or the community; rather, he was behaving more like a lurker consuming content he found interesting. Participant 96R was a pre-med student, and specifically went looking for discussions on Reddit related to his medical interests as well. As he found subreddits and discussions about topics related to what he was studying, he became excited reading the posts:

96R: "Parent's virus levels became undetectable after bone marrow therapy with stem cells." Stem cells are absolutely no. So we learned about them in Physiology and all the crazy stuff that they can do. How are they ethical in any type of way? I don't know. [... more talking about the content of the post ...]"

INTERVIEWER: So this is pertaining to your major, right?

96R "Heck, yeah. This is sweet.

Both participants who seek entertainment and participants who seek information expressed their interests as navigating the community. Few participants found content of the type they were looking for during the first attempt, so most of them needed to search or to browse around the online community to some extent.

\subsubsection{Appraising content}

Contrary to consumption, some participants explicitly tried to appraise and evaluate the content in the online community. 10 out of the 31 participants in our study were oriented toward appraising content at least once during the study.

As participants appraised content they had two simultaneous goals. First, they wanted to see if a certain type of information existed in the community. They often would have a particular topic or subject in mind, and use the search function or try to find a subreddit or Quora category to find the content. For example, not long after saying 'If I'm gonna post something on there, it'll probably be related to cars, yes.' (43R), participant 43R decided to search the site for information about cars

\footnotetext{
${ }^{9}$ Rooney is an English professional footballer.
} 
43R: Let's see lets search cars here, and let's see what turns up.

One participant(75R) had a perception that Reddit was not well supervised, and concluded that the content in Reddit is probably contentious and biased. To confirm his previous mental model, he searched for a controversial topic to see whether this original perception is correct:

75R: Well, I typed like [...] "white people". Yeah.

INTERVIEWER: Why did you type that?

75R Controversial. Somewhat, probably racist. [...] They open like a topic to group chat, and people can join and comment. These are controversial topics, and like I've never seen in other places before.

Second, participants who appraised content wanted to assess the quality of content to know whether the online community provide legitimate contents. That is, while appraising content, participants would read posts about topics that they already knew a lot about specifically in order to evaluate these posts for correctness. For example, participant 3Q had just completed a Masters in Public Health (MPH) degree, and she found a question on Quora about MPH degrees. After reading multiple answers, the interviewer asked her about them:

3Q: The responses were right. Whatever both people said is absolutely right about having an MPH. You don't need to be a doctor or have a $\mathrm{PhD}$, you can work with that degree, it's a degree in high demand.

After finding a satisfying answer from the community, 3Q spent more time navigating other contents that are not related to her professional knowledge. As conducting think-aloud while browsing new contents, 3Q became more engaged with content (i.e., consuming content); she reflected on her previous own experience, reading the following content;

3Q: Let's go back... To see...”What is it like to live in Williamston?".. (3Q read aloud texts)...I have worked at [company's name ${ }^{10}$ for six years, but I live in [city], 25 miles of Williamston, the commute is 15 minutes. I agree that Williamston is kind of a bore, and the winters can be pretty bad

\subsubsection{Appraising meta information}

A related method that 6 of the 31 participants used to appraise content based on meta information about the content, including the original source of content (if linked to a 3rd party website), timing of content, or votes on content. For example, participant $63 \mathrm{R}$ repeatedly looked at the source of linked information. Early during her focus on appraising content, she found content she wasn't sure about but then noticed 'Okay, it's taking me to the Wikipedia site, I guess. I don't know, maybe these are legit.' (63R) As she said a few minutes later,

${ }^{10}$ Information in brackets has been redacted to protect participant identity. 
63R: At first I just thought like it was just random people just posting things, and then when I clicked on the links like... Let's see, like this looks like a legit news post from somewhere.

Later she found another post with a link and concluded 'This is from the Washington Post, so this is obviously legit.' (63R).

This appraising of metadata also frequently led people to question content or the online community as a whole. Participant $62 \mathrm{R}$ questioned whether a specific post should be labeled "trending":

62R: I don't know how it can be trending if those were posted within eight hours. It's not that speedy with internet or whatever. I feel like it would have to be posted for longer than eight hours for it to be trending

Similarly, participant 43R questioned a post on Reddit based on voting results:

43R: I have no idea why these posts are listed as they are, because I don't think they're listed in the order of their popularity, because this one has less votes than this one

A number of votes on the contents helped participants judge whether the online community is working properly or to infer attributes about the community. For example, 45Q concluded that Quora is not a popular website since they only had 20 votes on the top question on the front page. His mental model to understand the popularity of the online community was formed by the degree of engagement with the top contents:

45Q: I don't know. There's only 20 votes on the top question on this page, upvotes, I'm assuming that is if you like something and you want it to be more popular and appear at the top, other websites like Flickr or something, you'll see 30,000 likes on a picture

Appraising meta information appeared to serve two goals. The meta information helped to directly appraise the content of the post and evaluate whether the post was valuable; this goal is very similar to the appraising content goals above. Second, appraising meta information helped to explicitly evaluate the mechanisms of the site and the online community as a whole.

\subsection{Orienting on people}

A second major area of focus for our participants was the people associated with the online community. 19 of our 31 participants looked into information related to the members themselves, such as their background or motivation for their contributions. These participants perceived online community members as individual agents whose interests and backgrounds determine the types of content available in the community, and specifically oriented their attention to conjecturing more 
about the people in the community. In this evaluation phase, they do not directly interact with existing members to learn norms or rules in the community, nor did they try to mimic experienced members' contribution.

When they oriented their attention to people, some participants (10 of 31) focused their attention on community members' information that are explicitly displayed on the site. For instance, participants took a look at users' profile to see their background. Particularly on Quora, many people focused on the explicitly provided author information. For example, participant 45Q described his confusion about why authors post on Quora based on explicitly provided labels:

45Q: I don't really know who... The kind of people that use this website, 'cause it says like... You know, like IT professional and entrepreneur... Like this guy, I think, analyst, author, historian... I don't know why they would be on here. I feel like they have better things to do.

Many of these subjects also expressed concern about not knowing the other members of the community. Participant 97Q felt like on Quora, 'It's just odd to answer someone's question, because you don't know that person' (97Q). These quick heuristic judgements about the people often led people to question the content in the community:

94Q: I think it lacks credibility with the people that are answering just 'cause I don't know. I don't know who these people are, what kind of credibility they have

While current users at Quora do not care about anonymity when they participate in the community (Paskuda and Lewkowicz 2015), the anonymous attributes made it difficult for potential new members to engage with the content.

Beyond the available information in terms of people, some other participants sought to make an inference about community members based on the content that they provided in the community. Three participants $(60 \mathrm{Q} / 77 \mathrm{R} / 48 \mathrm{Q})$ believed members in the community might have a professional background or enough knowledge in light of the content. They speculated about members' gender (48Q), occupation (75R), or age (96R). These inferences impacted participants' perspective on the community in either positive or negative ways. For example, participant 60Q believed Quora members are 'far knowledgeable, more than I would think for this type of environment' (60Q), which was seen as a positive. On the other hand, participant 48Q believed Quora wasn't for her because:

48Q: I feel it's really white dominated or something. And then, I feel like a lot Indian names or something or... So then I'm like maybe Asian people, too, or something. I guess, people in higher education maybe like graduate school level people or something. 'Cause I see like a lot of people with PhDs or something. 48Q thought her identity does not match the majority of users in the online community. 


\subsection{Orienting on technology}

The third major aspect of communities that our participants focused on was how the technology worked - both the user interface of the online community and underlying mechanisms (e.g., the up/down voting mechanism on Reddit) that drive and organize the community. 22 participants oriented their attention toward technology at least once during their exploration of the online community.

Technological tools in the online community helped novices carry out peripheral activities (Bryant et al. 2005). User interface and functional aspects of the community affected participants (i.e., potential new members) as well, but in a different way; those technological aspects influenced the construction of superficial understandings of the community or helped them envision their future behaviors, whereas legitimate peripheral participants learned practices or goals in the community through those technological tools. By observing system's states or any explanations of system, people are able to build a mental model that help them generate descriptions of system purpose and form (Rouse and Morris 1986). So we focused on how participants leverage technology components to understand the online community.

\subsubsection{User interface}

The participants were asked by the interviewer to comment on the interface of the online community as part of the interview protocol ("How is the appearance?" ). Thus, almost everyone made some comment about the features of the interface and how they reacted to it. However, 11 of our participants went beyond this, orienting toward the interface for a non-trivial period of time to try to understand how the interface shapes the community, by focusing on aspects of the interface beyond the superficial comments made by others. In this section, we summarize how these participants used the interface to better understand the online community they were looking at.

Some participants $(7 \mathrm{Q}, 48 \mathrm{Q}, 59 \mathrm{R}, 95 \mathrm{R}, 105 \mathrm{Q})$ were restrained by the first impression through the interface (i.e., a primacy effect). Their first impression dominated their expectation during the exploration of the online community. Even though they looked up some other artifacts in the community, their initial impression anchored their expectations. They just skimmed over detailed information in content, and instead looked at the superficial appearance. For instance, participant 59R described the interface of Reddit and its impact on his interest in the community:

59R: It looks kinda like, not dumbed down but it looks, what's the word I'm looking for? It's kinda like I'm looking at a whole bunch of stuff but I'm not really getting drawn to all the content on the website.

Aspects of the interface - such as color, fonts, or a grid - are considered as an important factor in evaluating the online community itself. Participant 7Q thought 
aesthetic aspects can make other people engage in Quora, which is an explicit acknowledgement of how technical design influences people's choices:

7Q: I mean I' $m$ a big into design, and I think the website is really kind of boring looking. Like there's not a ton of colors, and it's just like this one strip of words and columns... maybe the new design will get people interested.

\subsubsection{Mechanism}

Participants also focused on the online community's mechanism to understand how it is operated and built. Mechanisms are the functional or algorithmic aspects of the community that determine which content is displayed, and in what order. They are the technical affordances created to motivate or shape contributions, and interactions between features on the site. Mechanisms are explicitly sociotechnical aspects of an online community in that they are technical features or constraints that are put in place to shape and influence the behavior of members of the community. 12 participants oriented toward the mechanism during their exploration of the community, and only a few participants were influenced by the mechanism.

Often, participants who had no experience or any knowledge in the online community they were looking at would attempt to grasp the basic concept of the community by examining the mechanism and trying to explain the mechanism in their own words. As navigating the online community, participants understood what functions are available and how the online community lays out its contents (e.g., 'So let's see, top stories, questions, answers, blog posts are the options here. I'm just gonna go around a little bit. Open questions. Oh, there's a search bar at the top, as well.' (99Q), 'So this is how I picture anyone who down votes my posts. So I guess down votes means dislikes.' (28R)). They also guessed what was likely to happen if they chose to participate in the community by understanding the mechanism:

60Q: So the website is more like anyone can post an answer to any question, it looks like, and I can answer if I choose to

As navigating the community, some participants often became confused about how contents are sorted and how they are displayed on the page:

43R: I feel like all these posts should be categorized according to the topic, or heading that it should be under... I have no idea why these posts are listed as they are, because I don't think they're listed in the order of their popularity

62R: There is not really any organization of it, other than that has been posted recently, I guess. There are trending subreddits, except all of them are within the past eight hours.

We found that participants focused on the mechanism component, particularly when they found a mismatch between the way of how the online community 
displays content and the expectation of the participant. This mismatch often hindered participants from understanding the online community's concept and consuming its content.

\section{Findings: external influences}

When a person is evaluating an online community, they have very little information to go on. Potential new members are not situated in the social practices with existing members in the online community so that they rarely get help from experienced members directly and or indirectly. Due to this barrier, our participants relied on their previous experiences, external to the community, which are applied as critical criteria to evaluate the online community. As participants explored each of the different components of the online community, they also brought in information from friends about this community and about experiences with other communities via analogies.

\subsection{Friends and other sources}

Six participants were heavily influenced by things they had previously heard; most participants had a vague idea about Reddit or Quora but had not really heard of or visited them before. Participants then often based their orienting activities on the information they had previously heard. For example, participant 38R had an impression that Reddit was mostly for men:

INTERVIEWER: Have you heard about Reddit any other times other than that? 38R: Yeah, I mean, I feel like some of the guys I was friends with in undergraduate would talk about it and talk about going on it and posting on it and these kind of things. [...] It's been a while since I was an undergrad, so I don't remember exactly what they were saying about Reddit. I just remember that some of my male friends were involved in that.

As she browsed around the site, she concluded that Reddit 'seemed like a very male dominated space' (38R). As she evaluated the site, this initial, external perception gave her specific things to focus on and look at:

38R: from what I've seen, it does still seemed to be very male-dominated.

Even the comments in all the documentaries were fairly obviously from men, and a lot of the comments even on the women fitness' page were directed at defending men or these different issues

Similarly, participant 92R had heard from her brother that Reddit was 'a place where you can find things that Google doesn't find for you' (92R) and 'a place where you could ask more specific questions and potentially get well informed useful answers' (92R). This then directed her to look for environmental issues, which is a topic she was particularly interested in. 


\subsection{Analogies with other communities}

Many participants drew analogies with other communities or websites that they were more familiar with to understand the new-to-them community. They recalled their understandings of other communities, particularly when they attempted to make sense of how Reddit or Quora is operated and what they are for or to evaluate the quality of contents in comparison with other communities they already joined. In light of each observed component in the new community, participants were aware of unique or similar characteristic that allowed them to form the analogy. Analogical reasoning is a very common way to understand new situations (Klein 1998).

All but four participants made analogies with other communities at the same point during the interview session. As part of the interview protocol, the interviewer asked participants about their first impression after participants looked at the main page the first time ("What is your initial impression of the website?"). Ten participants made use of analogies to explain their first impression. In focusing on its appearance, they recalled other communities or websites which looked similar to Quora or Reddit.

Participants 60Q and 97Q scrutinized the mechanism — how it operates — by using their understanding of another community. They focused on not only on the overall interface but also on the basic concept of the community. Since both had no prior experience with Quora, they were more likely to spend time to understand it by using analogy:

97Q: Yeah, I mean, it's simple. It's not cluttered. [...] You can type in any question and the related topic and you can answer, right? I mean, these are like unknown people, right? Because I don't know any of them. It's just like Facebook, but with a larger community and with unknown people, with whom you can connect, I guess?

Most participants continued making analogies to expand their understanding, often comparing against multiple communities by recognizing the similar or unique components in those communities. After seeing similar content, some participants use analogies to clarify what type of content is available and differentiated its character from the analogized community:

96R: I guess. It brings you to a whole bunch of things that you didn't know necessarily was there, kind of like StumbleUpon... I feel Reddit has the most information, that's more... Has more variety than say, StumbleUpon or Imgur

Participant 43R enjoyed reading content on Reddit and found it to be similarly entertaining content on another community. His previous experiences in the other community (i.e., 9GAG) helped him judge whether contents in a new online community attract him: 'So, I feel that that's more straight humor, compared to like reading so much. There's post like these too on 9GAG, but like that's just way 
too much reading.' (43R). He continued then to try to distinguish the content on Reddit from the other community's content:

43R: 9GAG is just like browsing through pictures and names, and Reddit is for more reading I believe. 9GAG is like just for pictures, like the same difference between books and comics if you will.

\section{Findings: deciding about participation}

In this section, we focus on how a decision about whether to continue visiting the online community is made based on their initial mental models. As participants viewed the online community and talked about it with us, almost everyone focused on the central question of whether they wanted to continue participating in the community and if so, what kind of participation. Near the end of the interview, we explicitly asked our participants if they had made a decision and if so, what they decided. Some participants announced their opinions before we asked. 11 participants (35\%) indicated that they decided that they would be willing to join the community and participate. $13(42 \%)$ decided that the community was not for them and they did not want to continue participating. And the remaining 7 (23\%) indicated some form of "maybe", suggesting that they had not made up their mind or that they were unsure if they would continue participating.

As we mentioned earlier, there is little prior work focusing on potential new members who haven't decided to join in an online community. Therefore, we aim to illustrate the patterns we observed from our evidence rather than to generalize or confirm potential new members' decision process.

Each participant described motivations for their decision if they would participate in the online community. One pattern in these motivations was strong: people who are willing to join in the online community were predominantly influenced by the content of the community. People who didn't want to join had a wider variety of reasons for declining to engage further. Next, we present details about the role of each component in online communities and external influences in participants' decisions.

\subsection{Wanting to continue}

For both Reddit and Quora, the participants who found content they are interested in and enjoyed reading it are inclined to decide to continue visiting the community. When asked why they wanted to continue participating, they often described their satisfaction in the community since they were able to find a particular type of information that they liked. They mentioned that they wanted to continue coming back to the community to pursue more of that information. Participants, especially those who call themselves as heavy information seekers, frequently visiting many 
online communities and website to find new content, mentioned that they will revisit online communities:

96R: I learned is that it's not only for entertainment but you can learn a lot from it. I think that it just really opens you up to just a lot of information that you wouldn't normally regularly search yourself.

63R: I'd definitely use it just to like know information, just to know more information, just to know more about a topic. I'd probably just look, read. [...] I don't know how you say that, but it's science based and I like science so I'm always looking for new stuff to look into and stuff.

However, none of them started to be immersed in consuming content, rather most participants skimmed what content is available at the first. For this skimming content stage, participants rarely built clear mental models of the online communities to make a decision whether they are willing to join. However, our findings showed that this initial stage is important to scaffold an expectation that participants may be able to find interesting content if they explore the community with more time. For instance, after deciding to navigate more content (see details in 4.1.1), $2 \mathrm{R}$ spent time reading through different contents for a while, and then decided to revisit Reddit in the future:

2R: [After navigating Controversial tab] it's funny. But I kinda wanna see like what's under New. I think I just click New?... [talking aloud contents 2R paid attention] ...So that's cool. I'm probably not gonna read it alright now, 'cause I wanna look at more stuff. But I will probably save this to one of my favorites so I could read it later.

In making this decision, participants explicitly made an assumption that future content would continue to be very similar to what they could already see. Online communities are constantly evolving and changing, yet these participants would usually make the simplifying assumption that future content will be similar to past content.

Finding interesting content in the online community often took substantial amount of time, which might result in the failure to attract potential new members. Our findings indicated that other components in the community sometimes help participants find interesting content or to stick around the community. For instance, 27Q appreciated a recommendation function to make it easier to get relevant information:

27Q: Putting in the interest in the beginning, at least it's something that I'm interested in already. So I don't have go through random things, like doing thing like at Reddit. I just have to scroll till find something that looks interesting, but this thing has already got interesting things for me. 
Thanks to Quora's algorithm to sort out content users might be interested, 27Q was able to enjoy the serendipity of finding new information in the community:

27Q: I would definitely recommend it to certain people, especially people like me, they'd really like this kind of a website.[...] just the articles are there, on the topics you get to post what you think about this specific topic, and see what other people have to say about it. So kind of interesting.

After building a positive perception of people in the community (details in 4.2.2), 60Q also looked for more content to decide whether he wants to join in Quora. 60Q thought that it is worth trying to keep scrolling because people provided thoughtful responses rather than jokes or memes. In the end, he said 'It's interesting. I actually might use this now.' (60Q). Other components of online communities such as people and technology did not have an immediate impact on participants' decisions to revisit the community in the future. However, these components can be leveraged to promote more content consumption.

Our findings also showed that participants tended to make an allowance for drawback with other components if they put a priority on content. Participants were inclined to pass over some weaknesses in the community as long as they felt it was valuable for reading content. Usability, in particular, was a common complaint but not enough to prevent returning to the site. For instance, one person said 'I think overall, the usability of the website still bothers me' (52R), but still indicated that he wanted to return because he was interested in the content. 94Q was also not satisfied with particular aspects of the community (e.g., the anonymity of the community, the size of the community), but he decided to continue visiting the community, highlighting the value of the contents:

94Q: I think it needs to build up its community a little bit more. But, I think overall some of these questions are questions I would like to know the answer to.

The two communities studied - Quora and Reddit - specifically focus on and cater toward being general places for discussion and information. We believe it is not surprising that participants who were most interested in information seeking were the ones most likely to choose to continue participating as that interest matches the goals of the communities.

\subsection{Choosing not to continue}

On the other hand, participants who did not want to visit again usually formed their expectations by focusing on a wider variety of attributes of the online community. Participants cited content, people, and interface as reasons why they would not want to return.

Not everyone, but most participants who appraised the contents had an explicit goal: whether particular content is existing in the community. In this case, 
participants navigated the community more actively by using a function such as a search tool than those who enjoyed consuming content. However, they usually chose not to come back to the community. The main reason they want to check if Reddit or Quora has a particular content is that they want to compare them with the other online communities they are using:

INTERVIEWER: So moving on forward after doing this study, how do you think your use of Reddit will be in the future? Do you think it will be the same or will it change? ${ }^{11}$

43R: I don't think I'll change, now. I can search for cars on 9GAG and look at nice pictures of cars and stuff.

Participants who perceived Reddit or Quora as a similar type of online community that satisfies a common need they have gained from another online community were not inclined to join in new community (i.e., Reddit and Quora). For these participants, whether they are able to find different content is an important criterion for their decision; that is, they evaluated the community competitively against other communities, websites, and information sources in deciding if it was worth returning to. In contrast to 43R, 3Q wanted to revisit Quora in the future, because, for her Quora isn't a substitute of the other community she belongs too, but a complementary one that she can use:

3Q: It's not bad; it's interesting. It's different from like your cliche Google answers or Yahoo answers or Wikipedia answers. It's cool.

Another very clear attribute that discouraged participants in joining the community is the user interface, especially aesthetic aspects in the community. Users' first impressions of website aesthetics have a substantial influence on their opinions of the website (Reinecke et al. 2013). Our findings also showed that there appeared to be a primacy effect from the interface for a number of participants. The very first thing that people notice about a community is the interface and both of these communities had usability issues with their interfaces. Participants who, very early in their evaluation, focused on the interface and came away with a negative impression would often give vague "No" answers about whether they would be willing to return to the community. This clear mental model deterred participants with the content, rather they continuously critique other components (e.g., 48Q in section 4.2.2) to reinforce their negative attitude about the community. Some participants acknowledged that their decision not to revisit the community is derived from personal preference. The evaluation of aesthetic aspects in the user

\footnotetext{
11 Before Interviewer asked this question at the end of the interview, 43R already expressed his opinion that he may not be willing to revisit Reddit in the future
} 
interface might be subjective; some participants in our study preferred Quora's interface.

Similarly, strong preference for a certain attribute or strong previous perception sometimes dominated a process of understanding the new online community. For instance, the popularity of online community is an important criterion for $45 \mathrm{Q}$ in judging whether the community is attractive. As long as he realized that the Quora wasn't a popular community, he became disappointed in it and decided not to revisit:

45Q: I feel like if this were super popular, that would be something that I could get drawn into, 'cause it's not... I wouldn't feel like I was wasting my time necessarily.

The participant (38R) who had a negative perspective on Reddit did not change her attitude on the community and used the think-aloud session to confirm her previous mental model. As Kempton (1986) found earlier, no matter whether a participants' belief and understanding is correct or not, they tend to stick to their personal preference when interacting with technology. It showed that each participant has a different level of motivation or engagement based on their idiosyncrasies. Depending on the degree of motivation of how much they want to join in the community, the direct interaction with online community might not impact on people's decision.

Taken together, the most common conclusion that participants provided was that they saw few possibilities for them in the community based on their exploration. As they looked around, they formed expectations about how the community would be in the future. They wouldn't see many opportunities for their future participation for the different reasons as we noted above.

\section{Discussion}

In designing online communities, the needs of understanding potential new members are easily overlooked, even though online communities need to constantly attract new members (Kraut and Resnick 2012). One of main reasons to disregard the importance of potential new members is that capturing their behaviors to understand how they decide to join in the community is not simple. These non-member's behavior is not observable-reportable in the same way that others who have already been or signed up to join the community (Baumer et al. 2015). Online communities usually do not have their log data since they has not signed up for account yet, but what makes it more difficult is to know who they are. Each individual might have a different level of interest and motivations to join in the community, but technically anyone can possibly join in an online community if they are able to visit the community. To overcome these difficulty, our study attempted to explore how people, who heard about but haven't visited an 
online community, build their initial mental models and in what ways these initial understandings impact on their decision on whether they are willing to revisit the community. Our work is not confirmatory study, but exploratory one. We see the value of this study more as opening up possibilities and discussions in terms of potential new members as an important user type to build and maintain online communities. In this section, we will discuss how our findings extend prior work regarding new member recruitment (Kraut and Resnick 2012), considerations for recruiting potential new members, and the notion of participating in the online community.

\subsection{Understanding potential new member}

Our findings show that potential new members have unique patterns and goals in distinction from newcomers in the online community. Potential new members have a particular focus on evaluation to decide whether they want to continue interacting with the community. Each component in online communities and participants' previous perception functions as cues for developing their mental models to envision possibilities for joining in the community. During this evaluation phase, potential new members acknowledge that the online community is a socio-technical system and explicitly orient toward different aspects of the community one at a time to try to better understand the community and form a more complete mental model of it. This investigatory phase is distinguished with the learning phase where newcomers get involved with the legitimate peripheral activities in the online community. In this learning phase, newcomers get a sense of how a community actually works by participating in less important tasks or intentionally observing members' activities. On the other hand, our participants oriented toward socio-technical aspects of the community and applied their perception of the community to try to develop a better understanding of the community. For instance, they frequently skim content (and, especially, lists of content) not looking for things to look at in more detail, but to try to develop a big picture understanding of the types of content available on the site. They often look for and view content that they already know a lot about with the goal of assessing the content to determine if it is worth their time. And rather than interacting with other members in the community, participants frequently spent time trying to understand the types of people present in the community and understand their motivations for contributing content, because potential new members see these motivations as relevant for understanding what types of content will be contributed in the future. These investigatory behaviors can be considered as less formal and more cursory practices than legitimate peripheral participation. At this stage, potential new members' mental models, including underlying attitudes and beliefs, play a central role in moving forward to being involved in the community. 
This investigatory phase is a distinct phase of mental model formation, separate from lurking or non-use as well. In this phase, they are not exactly "lurkers" (Nonnecke and Preece 2003; Preece and Ben Schneiderman 2009) who are community members, but have made no visible contribution and regularly search for information (Muller et al. 2010). Some participants engaged in consuming content as if they were lurkers who are passively participating in the online community. However, participants did not go straight to finding and consuming content. Rather they usually evaluate other factors such as content availability, existing users' background, or the user interface before getting involved in contents. Also, they are not the explicit non-users that Baumer et al. (2013) describes because they have not yet made a decision about their use.

To recruit new members, retaining their participation and socializing them to get involved in the community's activities are important. However, individuals' first experiences in visiting the community are also critical to forming their expectations on their future participation in the community. These initial mental models can function whether they are willing to join in the community as a new member who will become socially engaged with the community's components. Given our evidence, in the investigatory phase (i.e., the first point of contact with the community), a way of interacting with online communities is relatively shallow, unstable, and sometimes parsimonious. Perhaps, potential new members may treat online communities like just like one of the websites they happen to visit in their everyday lives. It is certain that online communities are the place where people interact with others and engage in activities either directly or indirectly. On the contrary, each component in the community serves as a means to help potential new members evaluate the value of their efforts and time for revisiting. We argue that a way of understanding potential new members and their behavior shouldn't be done in the same manner as the new members or lurkers, whose experiences in the community are not their first.

\subsection{Considerations for design}

One of the most striking findings to us is that, among socio-technical components in the online community, the content was served as a strong material in forming positive attitudes about an online community when participants encountered the community. Our findings show that potential new members engaged in the community as absorbed in content, while they leveraged various components in the community to assess whether they are willing to join in the community. The more participants enjoyed consuming content (i.e., reading texts or any types of web content that online community's members have produced by leveraging technological assets in the community), the more likely they are willing to revisit the community. 
Previous work has highlighted the role of socio-technical components in the community as a means to incorporate new members to be situated in societal learning settings. Existing members in the online community are considered as a key resource to encourage new members to engage in the community. For instance, Kraut and Resnick (2012) suggest that providing opportunities to interact between newcomers and experienced users is beneficial for newcomers to get adjusted to the community. Communication between them would have a positive influence on the socializing process (Lampe and Johnston 2005; Yang et al. 2017). The functionality of technological tools in the online community would also be critical to facilitate the learning process. Particular tools in the user interface (e.g., search box, edit option), for instance, are exploited to nudge newcomers behaviors to participate in the peripheral activities (Bryant et al. 2005). For newcomers to become productive contributors, providing content such as a "how to" explanations - tools that allow newcomers to observe experts' practices or receive feedback from the older members in the community - is important. For instance, to assist the learning about online communities' norms and practices, (Mugar et al. 2014) introduced a concept of practice proxies. This is because it is assumed that these groups of users (i.e., newcomers) are already engaged in the community along with a sense of common purpose. However, potential new members are not mutually engaged in the community yet; instead, they are kind of bystanders to investigate the community. Our findings demonstrated that potential new members just want to refer to these components to build mental models of the community rather than to engage with existing members.

Our results inform that there is a need for further research on how to make use of the socio-technical components to successfully recruit more new members. Given our findings that people are more willing to join in the community when they are absorbed in the contents, one of the key elements is to make potential new members engage in the community is to switch their mode from an investigatory attitude to information seeking attitude. During the investigatory phase, potential new members attempt to acquire knowledge, which functions as an impetus for building or reshaping mental models. To support potential new members' investigatory phase, what needs to be considered in designing socio-technical components in the online community? In what way are potential new members engaging in content to seek information they are interested in? Mental models are consistently updated and developed over time, adapting to new information from the first experiences in the online community. How can designers employ these components in the online community to help potential new members form clear mental models and ultimately provoke positive attitudes to the community? We hope our findings can be served as a baseline to understand how to make potential new members engage in the community and further research needs to be done to help online communities successfully recruit broader populations. 


\subsection{The notion of participating in the online community}

The previous section discusses how potential new members' practices in the online community are distinguished from newcomers' ones. Here, we move on to reflect more broadly on the notion of participating in the online community. The first-time participation can be interpreted in different ways depending on whose perspective we put in focus. From an online community or community manager's point of view, participating in the community can be understood whether users took the first visible action such as sending a message to an existing member or leaving any comments or reactions on the community. If an online community has a login function, users can be considered a member if they signed up to the online community. In this case, participating in the community can be understood as being a member in the community no matter how much a user is involved with an community and its members. However, an individual's first experience within the community is not always the same as the first participation from the community's perspective (Seering et al. 2020).

Participants in our study, named as a potential new member, had their first and direct experience in the community during this study, but from the online community's perspective, their practices are not perceived as a first-time participation. Much research has been studied within the online community's perspective to discuss the challenge of recruiting new members (Kraut and Resnick 2012). By doing so, any type of new members (e.g., different visit count) can be considered as the same latent individual who can become an active member of the community. We argue that this dominant perspective in understanding new members may restrict considerations for recruiting tasks in the online community. According to our evidence, participants did not directly articulate whether they want to become a member of the community in the near future. Rather, they expressed their interests in the community and willingness to visit the community again as long as they said they will continue participating in the community. It indicates that from potential new members' perspective, participating in the online community does not necessarily mean being a member of the community.

In general, the central focus in HCI has been "using technology" rather than "not-using them" (Satchell and Dourish 2009). This dominant notion of "use" is also the main frame to conduct online community research, dealing with the challenge of recruiting new members. Therefore, the interaction between an individual user and socio-technical components in the online community (i.e., content, people, technology) is considered as the most important practice to understand new members' behaviors and to support them to become a legitimate member of the community. However, an individual user's first experience has received relatively less attention to recruiting new members. Our findings highlight that potential new users engage in a number of behaviors that are fairly specific and unique to achieve their own goals. With the dominant "using technology" approach, there should be a blind spot to understand users in a broad manner. Perhaps, a potential 
new member's experience in building mental models of the online community can be viewed as an instance of post-userism that "enables more comprehensive problem setting, facilitating a design scope" (Baumer and Brubaker 2017) to handle an issue of finding new members in an online community.

\section{Conclusion}

Online communities are a place where people can voluntarily visit and contribute. Recruiting new members is critical to sustaining an online community, and communities must be designed with that goal in mind (Kraut and Resnick 2012). While the importance of recruiting new members is often discussed, most studies focused on new members' socialization and retention stages, in which they are entangled with existing members, rather than their first experience in an online community. Understanding their first experience of the online community is important because their initial decision to revisit to or join in the community highly rests on their initial mental models formed during their first experience. So our study focused on a potential new members, who haven't yet engaged in an online community, but have potential to join as a new member, to identify how they first approach an online community to decide on whether they want to continue to visit the community. To do this, we conducted a qualitative interview study to investigate how people approach and evaluate online communities, and form mental models of the community. We found that people go through an explicit evaluation phase that is characterized by a distinct goal (evaluation) and distinct ways of interacting with the community that are different than how new members or lurkers participate in the community. Our data revealed that potential new members leveraged all socio-technical components in the online community and their previous perceptions on the community to build mental models of the online community. Since potential new members are not involved with any members in the community yet, a way of navigating the online community looks similar a way of navigating a general website rather than a typical online community where people interact with each other to achieve mutual or individual goals. Our findings urge that further research in terms of potential new members and their decision process in the long-term perspective is necessary for recruiting new members. We hope that designers will be inspired by our findings and will start closely investigate how potential new members develop their mental models for not just their first visit but also a longer term of their initial interaction to observe their decision process in more detail.

\section{Open Access}

This article is licensed under a Creative Commons Attribution 4.0 International License, which permits use, sharing, adaptation, distribution and reproduction in any medium or format, as long as you give appropriate credit to the original 
author(s) and the source, provide a link to the Creative Commons licence, and indicate if changes were made. The images or other third party material in this article are included in the article's Creative Commons licence, unless indicated otherwise in a credit line to the material. If material is not included in the article's Creative Commons licence and your intended use is not permitted by statutory regulation or exceeds the permitted use, you will need to obtain permission directly from the copyright holder. To view a copy of this licence, visit http:// creativecommonshorg/licenses/by/4.0/.

\section{Appendix A: Interview Protocol}

\section{A.1 Interview questions}

Before starting a think-aloud, we asked several questions to know participants' previous experience or knowledge about Quora/Reddit.

1. Can you tell me what your general understanding of Quora/Reddit is?

2. Before this study, when was the last time you heard about Quora/Reddit?

a) Can you tell me who mentioned Quora/Reddit to you?

b) Can you tell me what you were thinking about at that time?

c) Can you recall any other time when Quora/Reddit was mentioned to you?

3. In the past, can you please explain why you chose not to participate in Quora/Reddit?

4. Do you know anyone who uses Quora/Reddit?

5. What sort of things have you heard about Quora/Reddit in the past?

6. What are your thoughts or feelings about Quora/Reddit?

Note that we skipped questions 3, 5 and 6, if a participants had never heard or rarely knew about Quora/Reddit.

\section{A.2 An instruction and questions in think-aloud session}

In this portion of the study, I'll be asking you to complete simple tasks designed to help us evaluate Quora/Reddit. The most important thing for you to remember is that this is not a test - there is no right or wrong way to complete the exercises. The point of the study is to learn about why and how people choose to participate in online communities, and your honest responses will be extremely useful in helping us figure out this out.

In this session, I'm interested in two things: how you go about browsing the Quora/Reddit site, and what you think about as you do so. You'll be asked to 'think aloud'. It's very easy to do, imagine it as basically talking to yourself, but loud enough for me to hear.

When you are browsing Quora/Reddit, you will notice things that catch your attention. These things you see are important for our observation, so please verbalize these too. 
As you're doing the tasks, I won't be able to answer any questions. But if you do have questions, go ahead and ask them anyway so that I can learn more about what kinds of questions Quora/Reddit brings up. I'll answer your questions after the session.

You will have about five minutes to browse Quora/Reddit. Also, if you forget to think aloud, I'll say, 'Please keep talking.'

Do you have any questions about thinking aloud?

(Note: Subjects assigned in Quora were required to create an account on the site while the other subjects assigned Reddit were not required to do that. So we had a different guide between Quora and Reddit at the end of the think-aloud instruction.)

- Quora: You will first see the 'log-in' page. I would like you to make an account in order to access the Quora site. After which, you will begin setting up your account by picking your interests and then deciding whether or not to add 'friends' to participate. And finally, you will get to the main interface of Quora. I would like you to begin the 'think-aloud' at the 'log-in' page.

- Reddit: Before I take you to the home page of Reddit, I would like to show you Reddit's 'About' page. Then I will direct you to the home page. Once I hand you the computer, you may begin the 'Think-Aloud'.

Please browse the site and think aloud as you do so.

After taking a couple minutes to browse the site, what are your opinions about Quora/Reddit now? With your new knowledge of the site, would you participate in Quora/Reddit in the future?

Appendix B: Participants' Background

\begin{tabular}{llllr}
\hline Participant & $\begin{array}{l}\text { External } \\
\text { sources* }\end{array}$ & $\begin{array}{l}\text { Other com- } \\
\text { munities** }\end{array}$ & Major & Gender \\
\hline 2R & Y(By friends) & $\begin{array}{l}\text { Facebook, Twitter, } \\
\text { Instagram } \\
\text { Facebook, } \\
\text { Instagram, } \\
\text { GroupMe } \\
\text { Game } \\
\text { WeChat, forum, } \\
\text { Facebook, Twitter } \\
\text { Pinterest, }\end{array}$ & $\begin{array}{l}\text { Nutritional } \\
\text { Science } \\
\text { Public } \\
\text { Facebook }\end{array}$ & Fechanical \\
7Q & $\mathrm{N}$ & $\mathrm{F}$ & $\mathrm{F}$ \\
& $\mathrm{N}$ & $\begin{array}{l}\text { Hospitality } \\
\text { Management }\end{array}$ & $\mathrm{F}$ \\
& $\mathrm{N}$ & & &
\end{tabular}




\begin{tabular}{|c|c|c|c|c|}
\hline Participant & $\begin{array}{l}\text { External } \\
\text { sources* }\end{array}$ & $\begin{array}{l}\text { Other com- } \\
\text { munities** }\end{array}$ & Major & Gender \\
\hline $17 \mathrm{Q}$ & $\mathrm{Y}$ (By friends) & $\begin{array}{l}\text { WebMD, } \\
\text { answers.com } \\
\text { Facebook, } \\
\text { LinkedIn }\end{array}$ & Food Science & M \\
\hline $27 Q$ & $\mathrm{~N}$ & Reddit, Facebook & Economics & M \\
\hline $28 \mathrm{R}$ & Y(By friends) & $\begin{array}{l}\text { Facebook, } \\
\text { LinkedIn, Twitter }\end{array}$ & Communication & $\mathrm{F}$ \\
\hline $37 \mathrm{Q}$ & $\mathrm{N}$ & $\begin{array}{l}\text { Chinese online } \\
\text { Q\&A forum, Face- } \\
\text { book, Twitter, } \\
\text { Instagram, Tumblr }\end{array}$ & Business & $\mathrm{F}$ \\
\hline $38 \mathrm{R}$ & Y(By friends) & Tumblr, Facebook & $\begin{array}{l}\text { Sociology and } \\
\text { Environmental } \\
\text { studies }\end{array}$ & $\mathrm{F}$ \\
\hline $43 \mathrm{R}$ & Y(By friends) & 9GAG, Facebook & $\begin{array}{l}\text { Mechanical } \\
\text { Engineering }\end{array}$ & M \\
\hline $45 \mathrm{Q}$ & $\mathrm{N}$ & Wikipedia & $\begin{array}{l}\text { Politics and } \\
\text { Russian }\end{array}$ & M \\
\hline $47 \mathrm{R}$ & $x_{1}$ & Weibo, WeChat & $\begin{array}{l}\text { Finance and } \\
\text { Math }\end{array}$ & $\mathrm{F}$ \\
\hline $48 \mathrm{Q}$ & $\begin{array}{l}Y \text { (By search result } \\
\text { in Google) }\end{array}$ & $\begin{array}{l}\text { Tumblr, Pinterest, } \\
\text { Facebook, Youtube }\end{array}$ & $\begin{array}{l}\text { Economics } \\
\text { and } \\
\text { Psychology }\end{array}$ & $\mathrm{F}$ \\
\hline $52 \mathrm{R}$ & $\begin{array}{l}\text { Y (By social } \\
\text { media) }\end{array}$ & $\begin{array}{l}\text { Twitter, Instagram, } \\
\text { Facebook }\end{array}$ & Packaging & M \\
\hline $57 \mathrm{R}$ & $\begin{array}{l}\text { Y(By search result } \\
\text { in Google) }\end{array}$ & Facebook, Twitter & $\begin{array}{l}\text { Rehabilitation } \\
\text { counseling }\end{array}$ & $\mathrm{F}$ \\
\hline $59 \mathrm{R}$ & $\mathrm{N}$ & $\begin{array}{l}\text { LinkedIn, Face- } \\
\text { book, Twitter, } \\
\text { Instagram }\end{array}$ & Psychology & M \\
\hline $60 \mathrm{Q}$ & $\mathrm{N}$ & $\begin{array}{l}\text { Reddit, Face- } \\
\text { book, Twitter, } \\
\text { Technology } \\
\text { blog }\end{array}$ & Biochemistry & M \\
\hline $62 \mathrm{R}$ & Y (By friends) & $\begin{array}{l}\text { StumbleUpon, } \\
\text { Facebook, Twitter, } \\
\text { Instagram }\end{array}$ & $\begin{array}{l}\text { Human } \\
\text { Biology and } \\
\text { Spanish }\end{array}$ & M \\
\hline $63 R$ & $\mathrm{~N}$ & $\begin{array}{l}\text { Facebook, Twitter, } \\
\text { Instagram, Pinterest }\end{array}$ & $\begin{array}{l}\text { Kinesiology } \\
\text { and Communi- } \\
\text { cation }\end{array}$ & $\mathrm{F}$ \\
\hline
\end{tabular}




\begin{tabular}{|c|c|c|c|c|}
\hline Participant & $\begin{array}{l}\text { External } \\
\text { sources* }\end{array}$ & $\begin{array}{l}\text { Other com- } \\
\text { munities** }\end{array}$ & Major & Gender \\
\hline $71 \mathrm{R}$ & Y (By magazine) & $\begin{array}{l}\text { Facebook, } \\
\text { Twitter }\end{array}$ & $\begin{array}{l}\text { Media and } \\
\text { Informa- } \\
\text { tion }\end{array}$ & $\mathrm{F}$ \\
\hline $75 \mathrm{R}$ & $\begin{array}{l}\text { Y (By another } \\
\text { online forum) }\end{array}$ & $\begin{array}{l}\text { Academic forums, } \\
\text { Facebook, Youtube }\end{array}$ & Math & M \\
\hline $76 \mathrm{R}$ & $\mathrm{N}$ & $\begin{array}{l}\text { Hair forum, health } \\
\text { forum, Facebook }\end{array}$ & Social work & $\mathrm{F}$ \\
\hline 77R & $\begin{array}{l}\text { Y (By social } \\
\text { media) }\end{array}$ & $\begin{array}{l}\text { Facebook, Twitter, } \\
\text { Instagram }\end{array}$ & Marketing & $\mathrm{F}$ \\
\hline $92 \mathrm{R}$ & Y (By friends) & Facebook, Twitter & Sociology & $\mathrm{F}$ \\
\hline $94 \mathrm{Q}$ & $\mathrm{N}$ & $\begin{array}{l}\text { Facebook, Twitter, } \\
\text { Instagram }\end{array}$ & $\begin{array}{l}\text { Journalism } \\
\text { and Public } \\
\text { Relations }\end{array}$ & M \\
\hline $95 \mathrm{R}$ & $\mathrm{N}$ & $\begin{array}{l}\text { Facebook, Twitter, } \\
\text { Instagram }\end{array}$ & Social work & $\mathrm{F}$ \\
\hline $96 \mathrm{R}$ & Y (By friends) & $\begin{array}{l}\text { Facebook, Twitter, } \\
\text { Imgur, StumbleUpon }\end{array}$ & Neuroscience & M \\
\hline $97 \mathrm{Q}$ & $\mathrm{N}$ & Facebook & Marketing & $\mathrm{M}$ \\
\hline 99Q & $\mathrm{N}$ & Reddit & Neuroscience & M \\
\hline $103 R$ & $\mathrm{~N}$ & $\begin{array}{l}\text { Facebook, } \\
\text { LinkedIn, Twitter }\end{array}$ & Communication & M \\
\hline $105 \mathrm{Q}$ & $\mathrm{N}$ & $\begin{array}{l}\text { Facebook, } \\
\text { Instagram, Twitter }\end{array}$ & Business & M \\
\hline
\end{tabular}

- External sources*: If participants have heard about Reddit or Quora before, we fill in ' $\mathrm{Y}$ ' with the source. If not, we fill in ' $\mathrm{N}$ '.

- Other communities**: A list of any online communities or social media that participants have used before.

\section{References}

Antin, Judd; and Coye Cheshire (2010). Readers are not free-riders: Reading as a form of participation on wikipedia. In John Tang (ed.), CSCW '10: Proceedings of the 2010 ACM conference on Computer Supported Cooperative Work, Savannah, GA, USA, 6 - 10 February 2010. New York, NY, USA: Association for Computing Machinery, pp. 127-130.

Baumer, EricP.S.; Phil Adams; Vera D. Khovanskaya; Tony C. Liao; Madeline E. Smith; Victoria Schwanda Sosik; and Kaiton Williams (2013). Limiting, leaving, and (re)lapsing: an exploration of facebook non-use practices and experiences. In Strephen Brewster and Susanne Bødker (eds.), CHI '13: Proceedings of the SIGCHI Conference on Human Factors in Computing Systems, Paris, France, 27 April - 2 May 2013. New York, New York, USA: Association of Computing Machinery, pp. 3257-3266. 
Baumer, EricP.S.; Jenna Burrell; Morgan G. Ames; Jed R. Brubaker; and Paul Dourish (2015). On the Importance and Implications of Studying Technology Non-Use. In Interactions, vol. 22, 2, p. $52-56$.

Baumer, EricP.S.; and Jed R. Brubaker (2017). Post-userism. In Cliff Lampe; m.c. schraefel; Juan Pablo Hourcade; Caroline Appert; and Daniel Wigdor (eds.), CHI '17: Proceedings of the 2017 CHI Conference on Human Factors in Computing Systems, Denver, Colorado, USA, 6 - 11 May 2017. New York, New York, USA: Association for Computing Machinery, pp. 6291-6303.

Bryant, SusanL.; Andrea Forte; and Amy Bruckman (2005). Becoming Wikipedian: transformation of participation in a collaborative online encyclopedia. In Gloria Mark and Mark Ackerman (eds.), GROUP '05: Proceedings of the 2005 international ACM SIGGROUP conference on Supporting group work, Sanibel Island Florida USA, November, 2005. New York, NY, USA: Association for Computing Machinery, pp. 1-10.

Buntain, Cody; and Jennifer Golbeck (2014). Identifying social roles in reddit using network structure. In Andrei Broder; Kyuseok Shim; and Torsten Suel (eds.), WWW '14 Companion: Proceedings of the 23rd International Conference on World Wide Web, Seoul Korea, 7-11 April, 2014. New York, NY, USA: Association for Computing Machinery, pp. 615-620.

Burke, Moira; Cameron Marlow; and Thomas Lento (2009). Feed me: motivating newcomer contribution in social network sites. In Ken Hinckley; Meredith Ringel Morris; Scott Hudson; and Saul Greenberg (eds.), CHI '09: Proceedings of the SIGCHI Conference on Human Factors in Computing Systems, Boston, MA, USA, 4-9 April, 2009. New York, New York, USA: Association for Computing Machinery, pp. 945-954.

Butler, BrianS. (2001). Membership size, communication activity, and sustainability: A resourcebased model of online social structures. In: Information Systems Research, vol. 12, 4, pp. 346362.

Choi, Daejin; Jinyoung Han; Taejoong Chung; Yong-Yeol Ahn; Byung-Gon Chun; and Ted Taekyoung Kwon (2015). Characterizing Conversation Patterns in Reddit: From the Perspectives of Content Properties and User Participation Behaviors. In Rakesh Agrawal and Matthias Grossglauser (eds.), COSN '15: Proceedings of the 2015 ACM on Conference on Online Social Networks, Palo Alto, California, USA, November, 2015. New York, NY, USA: Association for Computing Machinery, pp. 233-243.

Anders Ericsson, K.; and Herbert A. Simon (1980). Verbal Reports as Data. In: Psychological Review, vol. 87, 3, pp. 215-251.

Eslami, Motahhare; Karrie Karahalios; Christian Sandvig; Kristen Vaccaro; Aimee Rickman; Kevin Hamilton; and Alex Kirlik (2016). First i like it, then i hide it: Folk theories of social feeds. In Cliff Lampe; Dan Morris; and Juan Pablo Hourcade (eds.), CHI '16: Proceedings of the 2016 CHI Conference on Human Factors in Computing Systems, San Jose, CA, USA. 7-12, May, 2017. New York, NY, USA: Association for Computing Machinery, pp. 2371-2382.

Fiesler, Casey; Shannon Morrison; R. Benjamin Shapiro; and Amy S. Bruckman (2017). Growing Their Own: Legitimate Peripheral Participation for Computational Learning in an Online Fandom Community. In ouise Barkhuus; Marcos Borges; and Wendy Kellogg (eds.), CSCW '17: Proceedings of the 2017 ACM Conference on Computer Supported Cooperative Work and Social Computing, Portland, Oregon, USA, 25 February - 1 March 2017. New York, NY, USA: Association for Computing Machinery, pp. 1375-1386.

Gelman, SusanA.; and Cristine H. Legare (2011). Concepts and folk theories. In Annual Review of Anthropology, vol. 40, pp. 379-398.

Halasz, FrankG.; and Thomas P. Moran (1983). Mental models and problem solving in using a calculator. In Raoul N. Smith and Richard W Pew (eds.), CHI '83: Proceedings of the SIGCHI Conference on Human Factors in Computing Systems. New York, NY, USA: Association for Computing Machinery, pp. 212-216. 
Jackson, Corey; Carsten Østerlund; Veronica Maidel; Kevin Crowston; and Gabriel Mugar (2016). Which Way Did They Go?: Newcomer Movement through the Zooniverse. In Pernille Bjørn and Joseph Konstan (eds.), CSCW'16: Proceedings of the 19th ACM Conference on ComputerSupported Cooperative Work and Social Computing, San Francisco, USA, 27 February - 2 March, 2016. New York, New York, USA: Association for Computing Machinery, pp. 624-635.

Johnson, ChristopherM. (2001). A survey of current research on online communities of practice. In: The internet and higher education, vol. 4, 1, pp. 45-60.

Jones, NatalieA.; Helen Ross; Timothy Lynam; Pascal Perez; and Anne Leitch (2011). Mental Models : An Interdisciplinary Synthesis of Theory and Methods. In: Ecology and Society, vol. 16, 1, pp. 1-13.

Kang, Ruogu; Laura Dabbish; Nathaniel Fruchter; and Sara Kiesler (2015). "My Data Just Goes Everywhere:" User Mental Models of the Internet and Implications for Privacy and Security. In Robert Biddle and Sunny Consolvo (eds.), SOUPS '15: Proceedings of the Eleventh Symposium On Usable Privacy and Security, Ottawa, Canada, 22-24 July, 2015. USENIX Association, pp. $39-52$.

Kempton, Willett (1986). Two theories of home heat control. In: Cognitive Science, vol. 10, 1, pp. 7590.

Kincaid, Jason (2010). Quora's Highly Praised Q\&A Service Launches To The Public (And The Real Test Begins). TechCrunch. https://techcrunch.com/2010/06/21/quoras-highly-praised-qaservice-launches-to-the-public-and-the-real-test-begins/.

Klein, GaryA. (1998). Sources of Power: How People Make Decisions. How People Make Decisions. Cambridge, MA, USA: MIT Press.

Kraut, Robert E.; and Paul Resnick (eds) (2012). Building Successful Online Communities: Evidence-Based Social Deisgn. Cambridge, MA, USA: MIT Press.

Lampe, Cliff; and Erik Johnston (2005). Follow the (slash) dot: effects of feedback on new members in an online community. In Gloria Mark and Mark Ackerman (eds.), GROUP '05: Proceedings of the 2005 international ACM SIGGROUP conference on Supporting group work, Sanibel Island Florida USA, November, 2005. New York, NY, USA: Association for Computing Machinery, pp. 11-20.

Lave, Jean; and Etienne Wenger (1991). Situated learning: Legitimate peripheral participation. Cambridge University Press.

Lu, Di; and Rosta Farzan (2015). Time to Introduce Myself!: Impact of Self-disclosure Timing of Newcomers in Online Discussion Forums. In Pete Burnap and Susan J Halford (eds.), WebSci '15: Proceedings of the ACM Web Science Conference, Oxford United Kingdom, June 2015. New York, New York, USA: Association for Computing Machinery, pp. 1-9.

Lu, Di; Rosta Farzan; and Claudia Lopez (2017). To Go or not to Go!: What Influences Newcomers of Hybrid Communities to Participate Offline. In Ingrid Mulder (ed.), $C \& T$ '17: Proceedings of the 8th International Conference on Communities and Technologies, Troyes, France, 26-30 June, 2017. New York, New York, USA: Association for Computing Machinery, pp. 159-168.

Miles, MatthewB.; A. Michael Huberman; and Johnny Saldaña (2019). Qualitative Data Analysis. A Methods Sourcebook. Sage Publishing, fourth edn.

Muller, Michael; N. Sadat Shami; David R. Millen; and Jonathan Feinberg (2010). We are all lurkers: consuming behaviors among authors and readers in an enterprise file-sharing service. In Tom Gross and Madhu Reddy (eds.), GROUP '10: Proceedings of the 16th ACM international conference on Supporting group work, Sanibel, FL, USA, 7 - 10 November 2010. New York, NY, USA: Association for Computing Machinery, pp. 201-210.

Muller, Michael (2012). Lurking as personal trait or situational disposition: lurking and contributing in enterprise social media. In Jonathan Grudin; Gloria Mark; and John Reidl (eds.), 
CSCW'12: Proceedings of the ACM 2012 conference on Computer Supported Cooperative Work, Seattle Washington USA, 11-15 February, 2012. New York, New York, USA: Association for Computing Machinery, pp. 253-256.

Mugar, Gabriel; Carsten Østerlund; Katie DeVries Hassman; Kevin Crowston; and Corey Brian Jackson (2014). Planet hunters and seafloor explorers: legitimate peripheral participation through practice proxies in online citizen science. In Meredith Ringel Morris and Madhu Reddy (eds.), CSCW'14: Proceedings of the 17th ACM conference on Computer supported cooperative work and social computing, Baltimore, Maryland, USA, 15 - 19 February, 2014. New York, NY, USA: Association for Computing Machinery, pp. 109-119.

Nonnecke, Blair; and Jenny Preece (2003). Silent Participants: Getting to Know Lurkers Better. In Christopher Lueg and Danyel Fisher (eds.), From Usenet to CoWebs, London: Springer, Computer Supported Cooperative Work, chap. 6. pp. 110-132.

Norman, DonaldA. (1983). Some observations on mental models. In Diedre Gentner and Albert L. Stevens (eds.), Mental models, New York, NY, USA: Psychology Press, chap. 1. pp. 15-22.

Norman, DonaldA. (2013). The design of everyday things: Revised and expanded edition. Basic Book, revised edn.

Onwuegbuzie, AnthonyJ.; and Nancy L. Leech (2007). Validity and qualitative research: an oxymoron? In Quality \& Quantity, vol. 41, 2, pp. 233-249.

Panciera, Katherine; Aaron Halfaker; and Loren Terveen (2009). Wikipedians are born, not made: a study of power editors on Wikipedia. In Wolfgang Prinz and Wayne G. Lutters (eds.), GROUP '09: Proceedings of the ACM 2009 international conference on Supporting group work, Sanibel Island, FL, USA, 10 - 13 May, 2009. New York, New York, USA: Association for Computing Machinery, pp. 51-60.

Panciera, Katherine; Reid Priedhorsky; and Thomas Erickson (2010). Lurking? cyclopaths?: a quantitative lifecycle analysis of user behavior in a geowiki. In Geraldine Fitzpatrick; Scott Hudson; Keith J Edwards; and Tom Rodden (eds.), CHI '10: Proceedings of the SIGCHI Conference on Human Factors in Computing Systems, Atlanta, GA, USA, 10 - 15 April, 2010. New York, NY, USA: Association for Computing Machinery, pp. 1917-1926.

Paskuda, Malte; and Myriam Lewkowicz (2015). Anonymous Quorans are still Quorans, just anonymous. In Fiorella De Cindio and Volkmar Pipek (eds.), $C \& T$ '15: Proceedings of the 7th International Conference on Communities and Technologies, Limerick, Ireland, 27 - 30, June, 2015. New York, New York, USA: Association for Computing Machinery, pp. 9-18.

Paul, SharodaA.; Lichan Hong; and Ed H. Chi (2012). Who is Authoritative? Understanding Reputation Mechanisms in Quora. arXiv:1204.3724.

Preece, Jenny; and Ben Schneiderman (2009). The Reader-to-Leader Framework: Motivating Technology-Mediated Social Participation. In: AIS Transactions on Human-Computer Interaction, vol. 1, 1, pp. 13-32.

Priedhorsky, Reid; and Mikhil Masli (2010). Eliciting and focusing geographic volunteer work. In John Tang (ed.), CSCW '10: Proceedings of the 2010 ACM conference on Computer supported cooperative work, Savannah, GA, USA, 6-10 February, 2010. New York, New York, USA: Association for Computing Machinery, pp. 61-70.

Reinecke, Katharina; Tom Yeh; Luke Miratrix; Rahmatri Mardiko; Yuechen Zhao; Jenny Liu; and Krzysztof Z. Gajos (2013). Predicting Users' First Impressions of Website Aesthetics with a Quantification of Perceived Visual Complexity and Colorfulness. In: Proceedings of the SIGCHI Conference on Human Factors in Computing Systems, Paris, France, 27 April - 2 May 2013. New York, NY, USA: Association for Computing Machinery, p. 2049-2058.

Rouse, WilliamB.; and Nancy M. Morris (1986). On looking into the black box: Prospects and limits in the search for mental models. In Psychological bulletin, vol. 100, 3, pp. 349-363.

Satchell, Christine; and Paul Dourish (2009). Beyond the User: Use and Non-Use in HCI. In: Proceedings of the 21st Annual Conference of the Australian Computer-Human Interaction Special 
Interest Group: Design: Open 24/7, Melbourne, Australia, 23 - 27 November, 2009. New York, NY, USA: Association for Computing Machinery, OZCHI '09, p. 9-16.

Seering, Joseph; Jessica Hammer; Geoff Kaufman; and Diyi Yang (2020). Proximate Social Factors in First-Time Contribution to Online Communities. In Joanna McGrenere; Andy Cockburn; Ignaci (Nacho) Avellino; Alix Goguey; Pernille Bjørn; Shengdong (Shen) Zhao; Briane Paul Samson; and Rafal Kocielnik (eds.), CHI '20: Proceedings of the 2020 CHI Conference on Human Factors in Computing Systems, Honolulu, HI, USA, 25 - 30 April, 2020. New York, NY, USA: Association for Computing Machinery, pp. 1-14.

Solomon, Jacob; and Rick Wash (2014). Human-What Interaction? Understanding User Source Orientation:. In: HFES '14: Proceedings of the Human Factors and Ergonomics Society Annual Meeting. Los Angeles, CA, USA: SAGE Publications, vol. 58, pp. 422-426.

Sundar, S.Shyam; and Clifford Nass (2000). Source Orientation in Human-Computer Interaction: Programmer, Networker, or Independent Social Actor. In: Communication Research, vol. 27, 6, pp. 683-703.

Velasquez, Alcides; Rick Wash; Cliff Lampe; and Tor Bjornrud (2014). Latent users in an online user-generated content community. In: Computer Supported Cooperative Work (CSCW), vol. 23, 1, pp. 21-50.

Wang, Gang; Konark Gill; Manish Mohanlal; Haitao Zheng; and Ben Y. Zhao (2013). Wisdom in the social crowd: an analysis of quora. In Ricardo Baeza-Yates and Sue Moon (eds.), $W W W$ '13: Proceedings of the 22nd international conference on World Wide Web, Rio de Janeiro Brazil, May, 2013. New York, NY, USA: Association for Computing Machinery, pp. 1341-1352.

Wash, Rick (2010). Folk models of home computer security. In Lorrie Faith Cranor (ed.), SOUPS '10: Proceedings of the Sixth Symposium on Usable Privacy and Security, Redmond, WA, USA, 14-16 July, 2010. New York, New York, USA: Association for Computing Machinery, pp. 1-16.

Weninger, Tim; Xihao Avi Zhu; and Jiawei Han (2013). An exploration of discussion threads in social news sites: A case study of the reddit community. In: ASONAM 2013: IEEE/ACM International Conference on Advances in Social Networks Analysis and Mining, Niagara Falls, Canada, 25-28 August, 2013. IEEE, pp. 579-583.

Yang, Lei; and Xavier Amatriain (2016). Recommending the World's Knowledge: Application of Recommender Systems at Quora. In Shilad Sen (ed.), Proceedings of the 10th ACM Conference on Recommender Systems, Boston MA USA, 15 - 19 September, 2016. New York, NY, USA: Association for Computing Machinery, pp. 389-389.

Yang, Diyi; Robert Kraut; and John M. Levine (2017). Commitment of newcomers and old-timers to online health support communities. In: Proceedings of the 2017 CHI conference on human factors in computing systems, Denver, CO, USA, 6 - 11 May, 2017. New York, NY, USA: ACM, pp. 6363-6375.

\section{Publisher's Note}

Springer Nature remains neutral with regard to jurisdictional claims in published maps and institutional affiliations. 